\title{
RETINOBLASTOMA RELATED (RBR) interaction with key factors of the RNA- directed DNA methylation (RdDM) pathway.
}

\author{
León-Ruiz Jesús ${ }^{1+}$; Espinal-Centeno Annie ${ }^{1+}$; Blilou Ikram²; Scheres Ben³ ${ }^{2}$ Arteaga-Vázquez Mario**
} and Cruz-Ramírez Alfredo ${ }^{1 *}$.

1. Laboratory of Molecular and Developmental Complexity at Laboratorio Nacional de Genómica para la Biodiversidad, Centro de Investigación y de Estudios Avanzados del Instituto Politécnico Nacional, (CINVESTAV-IPN), 36590 Irapuato, México.

2. Biological and Environmental Science and Engineering Division (BESE), King Abdullah University of Science and Technology (KAUST), Thuwal, 23955 Saudi Arabia.

3. Laboratory of Molecular Biology, Department of Plant Sciences, Wageningen University, Wageningen, the Netherlands.

4. Group of Epigenetics and Developmental Biology, Instituto de Biotecnología y Ecología Aplicada (INBIOTECA), Universidad Veracruzana, 91090, Xalapa, México.

+ These authors contributed equally to this work

*Authors for correspondence: alfredo.cruz@cinvestav.mx, maarteaga@uv.mx

\section{Summary}

- Transposable elements and other repetitive elements are silenced by the RNAdirected DNA methylation pathway (RdDM). In RdDM, POLIV-derived transcriptsare converted into double stranded RNA (dsRNA) by the activity of RDR2 and subsequently processed into 24 nucleotide short interfering RNAs (24-nt siRNAs) by DCL3. 24-nt siRNAs are recruited by AGO4 and serve as guides to direct AGO4siRNA complexes to chromatin bound POLV-derived transcripts generated fromthe template/target DNA. The interaction between POLV, AGO4, DMS3, DRD1, RDM1 and DRM2 promotes DRM2-mediated de novo DNA methylation.

- The Arabidopsis Retinoblastoma protein homolog is a master regulator of cellcycle, stem cell maintenance and development. In silico exploration of RBR protein partners revealed that several members of the RdDM pathway contain a motif that confers high affinity binding to RBR, including the largest subunits of POLIV and POLV (NRPD1 and NRPE1), the shared second largest subunit of POLIV and POLV (NRPD/E2), RDR1, RDR2, DCL3, DRM2 and SUVR2. We demonstrate that RBR binds to DRM2, DRD1 and SUVR2. We also report that seedlings from loss-offunction mutants in RdDM and in $R B R$ show similar phenotypes in the root apical meristem. Furthermore, we show that RdDM and SUVR2 targets are up-regulated in the 35S::AmiGO-RBR background.

- Our results suggest a novel mechanism for RBR function in transcriptional gene silencing based on the interaction with key players of the RdDM pathway and opens several new hypotheses, including the convergence of RBR-DRM2 on the transcriptional control of TEs and several cell/tissue and stage-specifictargetgenes.

Keywords: RdDM, de novo DNA methylation, RETINOBLASTOMA, Development, epigenetics.

\section{Introduction}


DNA methylation is essential for proper development in eukaryotes. In plants, it is involved in the regulation of gene expression, the defense against invasive nucleic acids, both of them with effects on development and physiology. In plants, cytosines can be methylated in symmetrical (CG or $\mathrm{CHG}$ ) and asymmetrical $(\mathrm{CHH})$ sequence contexts (where $\mathrm{H}$ can be $\mathrm{A}$, $\mathrm{T}$, or $\mathrm{C}$ ). Transposable elements (TEs) and other repetitive sequences are the main targets of DNA methylation (Borges and Martienssen, 2015; Matzke and Mosher, 2014). The major small RNA-mediated epigenetic pathway involved in de novo DNA methylation is the RNA-directed DNA methylation (RdDM) pathway (Matzke and Mosher, 2014; Erdmann and Picard, 2020). RdDM involves the function of Nuclear RNA Polymerase D (NRPD) or POL IV and NRPE or POLV (Hagg and Pikaard, 2011). POLIV transcribes short single stranded RNA (ssRNA) 26 to 45 nt in length (from the target locus that will be methylated) that serve as substrate for RNA-DEPENDENT RNA POLYMERASE 2 (RDR2) for the generation of double stranded RNA (dsRNA). The resulting dsRNA is processed by DICER-LIKE 3 (DCL3) into 24-nt small interfering RNAs (siRNAs). HUA ENHANCER 1 (HEN1) methylates 24-nt siRNAs at their 3'-end and are subsequently recruited by ARGONAUTE 4 (AGO4) (or other close paralog such as AGO6 and AGO9). The AGO4-siRNA complex associates with chromatin bound POLV-dependent transcripts produced from the same loci that will be methylated, through RNA-RNA pairing. The association between the AGO4-siRNA complex and POLV is further stabilized by protein-protein interactions between AGO4 and the CTD of POLV. Recruitment of the de novo DNA methyltransferase DOMAINS REARRANGED 2 (DRM2) to the template/target DNA occurs through the activity of RNA-DIRECTED DNA METHYLATION 1 (RDM1) that is able to bind methylated single stranded DNA (ssDNA) and also interacts with DRM2 and AGO4 (reviewed in Matzke \& Mosher, 2014; Trujillo et al., 2018).

Retinoblastoma proteins are multi-faceted master regulators of cell reprogramming in eukaryotes and are involved in the control of cell cycle, DNA damage response and in protein-protein interactions (PPIs) with transcription factors that modulate stem cell maintenance and asymmetric cell division for proper cell lineage commitment (Calo et al., 2010; Cruz-Ramirez et al., 2012; Harashima \& Sugimoto, 2016; reviewed in Dyson, 2019; reviewed in Desvoyes \& Gutiérrez, 2020). In Arabidopsis, RETINOBLASTOMA RELATED (RBR) has been shown to bind DNA, putatively to regulate the transcription of hundreds of genes and transposable elements (Bouyer et al., 2018) and also indirectly modulates gene expression by PPls and genetic interactions with lineage-specific transcription factors (CruzRamirez et al., 2012; Cruz-Ramirez et al., 2013; Matos et al., 2014; Zhao et al., 2017), chromatin-remodeling factors such as PICKLE (PKL) (Ötvös et al., 2021), and the Polycomb Repressor Complex 2 (PRC2) (Julien et al., 2018). The PRC2 complex regulates plant growth and development through the trimethylation of Lysine 27 on Histone 3 (H3K27me3), a well-known epigenetic mark involved in transcriptional repression. Two independent studies have established the connection between RBR and PRC2. Jullien et al., (2008) demonstrated that RBR directly binds to 
MULTICOPY SUPPRESSOR OF IRA1 (MSI1), an essential component of Arabidopsis PRC2 protein complexes involved in female gametogenesis, seed and vegetative development. The RBR-MSI1 complex directly represses DNA METHYLTRANSFERASE 1 (MET1) transcription, MET1 is a DNA methyltransferase acting on cytosine methylation at symmetrical $\mathrm{CpG}$ positions. MET1 repression occurs only on the female gamete and is required for the expression of imprinted genes. A similar observation was also reported by Johnston et al. (2008). The interaction between RBR and PRC2 is potentially deeper since FERTILIZATIONINDEPENDENT ENDOSPERM (FIE), another member of the PRC2 complex that interacts with MEDEA (MEA), SWINGER (SWN) and CURLY LEAF (CLF) (Oliva et al., 2016) does contain a highly conserved LXCXE motif, which is characteristic of proteins that bind with high-affinity to RBR ( Cruz-Ramírez, et al., 2012).

Plant and animal Retinoblastoma proteins share conserved residues that allow them to interact with proteins containing an LxCxE SLiM (SLiM: Short Linear Motif) RBR-binding motif (Lee et al., 1998, Dick, 2007). A decade ago, a global search in the Arabidopsis proteome for proteins containing the LxCxE SLiM led us to the identification of hundreds of candidates that potentially interact with the single Arabidopsis Retinoblastoma protein: RBR. By employing the LxCxE motif, that confers high-affinity to RBR, as an in silico bait to identify Arabidopsis RBR protein partners (Cruz-Ramirez et al., 2012), we identified several components of the RdDM pathway including the largest subunits of POLIV and POLV, RDR1, RDR2, DCL3, DRM2 and SUVR2 as potential targets of RBR. In this study we demonstrate that RBR binds to DRM2, DRD1 and SUVR2. We also report that seedlings of loss-offunction mutants in RBR and in genes of the RdDM pathway show phenotypes in the root apical meristem, with defects in the RSCN. This is consistent with the observation that RdDM and SUVR2 targets are up-regulated when RBR is posttranscriptionally silenced using the cell-type-specific artificial microRNA for Genesilencing Overcome (amiGO) system. Our results uncover a novel mechanism for RBR function in transcriptional silencing through its interactions with key components of the RdDM pathway and opens the possibility of a convergent action of RBR-DRM2 in the regulation of TEs and lineage or tissue-specific transcription factors, and stem cell regulators, such as WUSCHEL, AGL15 and POLAR, among other interesting putative target genes. 
122

123

124

125

126

127

128

129

130

131

132

133

134

135

136

137

138

139

140

141

142

143

144

145

146

147

148

149

150

151

152

153

154

155

156

157

158

159

160

161

162

163

164

\section{Materials and Methods}

\section{Plant Materials}

Arabidopsis thaliana plants were grown as described in Cruz-Ramirez et al. (2004). Col-0 wild type, double (nrpd2a-2;nrpd2b-1) and triple mutants (drm1;drm2;cmt3) plants were used for phenotypic analyses, as well as transgenic lines (pRBR::RBR:CFP, pDRM2::DRM2-GFP and 35S::AmiGORBR) (Cruz-Ramirez et al., 2012; Cruz-Ramirez et al., 2013).

\section{Microscopic Analysis}

Seedlings were grown and roots were prepared for confocal microscopy as previously described (Cruz-Ramirez et al., 2012). Fluorescent signals for the diverse genetic backgrounds were recorded with a Leica SP2 CLSM and a Zeiss LSM 800 CLSM. Roots were mounted and stained with Lugol as in Willemsen et al. (1998) and were visualized by Nomarski optics.

\section{Protein-Protein interaction (PPI) assays}

Yeast two-hybrid $(\mathrm{Y} 2 \mathrm{H})$ interactions were characterized by employing the ProQuest Two-Hybrid System (Invitrogen Life Technologies) as reported in Cruz-Ramirez et al., (2013). To quantify the strength of each interaction, three biological and technical replicates of Beta-galactosidase assays with CPRG as substrate were performed. Bimolecular Fluorescence Complementation Assays in Arabidopsis protoplast were performed as reported in Cruz-Ramirez et al., (2012). For RBR-DRM2, RBR-DRD1 and controls YFP fluorescence was recorded with a Leica SP2 CLSM.

\section{Computational analyses and ortholog identification}

Angiosperm protein sequences were downloaded from Phytozome (https://phytozome-next.jgi.doe.gov/), while non-angiosperm and algae protein sequences were downloaded from Phytozome, Fernbase (Li et al., 2018), TreeGenes (Wegrzyn et al., 2019) and Phycocosm (Grigoriev et al., 2021). Sequences for $A$. agrestis and $P$. margaritaceum were downloaded directly from the University of Zurich Hornworts database (Li et al., 2020: https://www.hornworts.uzh.ch/en.html) and the Penium genome database (Jiao et al., 2020: http://bioinfo.bti.cornell.edu/cgi-bin/Penium/blast.cgi), respectively. LXCXE-SLiM containing protein sequences were detected using a custom perl script (Caballero-Perez, personal communication). To infer orthologues, all protein sequences from all 28 species analyzed were placed into orthogroups using the OrthoFinder software (Emms et al., 2019). 


\section{qRT-PCR assays of RdDM targets}

Twenty seedlings of 14-days-old post germination plants from Col-0 or 35S::AmiGORBR (Cruz-Ramírez et al., 2013), were used for total RNA extraction by TRIzol reagent (ThermoFisher) in three biological replicates. Total RNA was used to generate cDNAs according to the manufacturer's protocol for SuperScript III (ThermoFisher) we used $5 \mu \mathrm{g}$ of total RNA per $20 \mu \mathrm{L}$ reaction. The expression level was determined using SYBR GREEN mix (ThermoFisher) in a $10 \mu \mathrm{L}$ reaction. The data were normalized using Actin 7 expression levels. The primers used in these experiments are those reported in Han et al. (2014).

\section{Results and discussion}

\section{Major players of the RdDM pathway and their putative RBR-Binding motifs}

Early predictions for Arabidopsis RBR-interactors, served as the basis for the functional characterization of the interaction between RBR with diverse lineagespecific factors such as SCARECROW, FAMA, XND1 and PICKLE, among others (Cruz-Ramirez et al., 2012; Matos et al., 2014; Zhao et al., 2017; Zhou et al., 2019; Ötvös, et al., 2021). In addition to the aforementioned proteins, we identified many proteins with diverse key molecular and cellular functions bearing the RBR-binding motif which, in many cases, were evolutionarily conserved. Among them, we found that components of the RdDM pathway including NRPD2, DRD1, DRM2, DCL3 and SUVR2 contain the canonical LxCxE SLiM (Fig. 1, TableS1). We also found that major players of the RdDM pathway including NRPD1, NRPE and RDR2 contain a non-canonical RBR-interaction motif VLxFxE (Fig. 1, TableS1). The observation that eight components of the RdDM pathway shared canonical and non-canonical RBRinteraction motifs prompted us to investigate if some of them are true physical RBR interactors. 


\section{Conservation of LxCxE-like motifs in RdDM factors along Viridiplantae}

195

196

197

198

199

200

201

202

203

204

205

206

207

208

209

210

211

212

213

214

215

216

217

218

219

220

221

222

223

224

225

226

227

228

229

230

231

232

233

To gain insight into the evolutionary conservation of the LXCXE SLiM present in components of the RdDM pathway, we interrogated publicly available plant and algae genomes aiming to detect the presence of canonical and non-canonical (IXCXE/LxCXD/IxCXD) LxCXE SLiMs among orthologs of the RdDM pathway along the Viridiplantae clade. To optimize the breadth of the plant phylogeny to cover, we focused on a small subset of species with available sequenced genomes representing each major lineage of the Viridiplantae kingdom. The species selected and analyzed include representatives from angiosperms ( $A$. thaliana [Ath]; Boechera stricta [Bsf]; Solanum lycopersicum [Sly]; Zea mays [Zma]; Setaria viridis [Sv]]; Oryza sativa [Osa]; Amborella trichopoda [Atr]), gymnosperms (Picea abies [Pab]; Pinus taeda [Pta]; Gnetum montanum [Gma]), ferns (Azolla filliculoides [Af]; Salvinia cucullata [Scu]; Ceratopteris richardii [Cri]), lycophytes (Selaginella moellendorffii [Smo]), bryophytes (Sphagnum fallax [Sfa]; Physcomitrium patens [Ppa]; Marchantia polymorpha [Mpo]; Anthoceros agrestis [Aag]) charophyte (Penium margaritaceum [Pma]; Mesotaenium endlicheranium [Men]; Spirogloea muscicola [Smu]; Chara braunii [Cbr]; Klebsormidium nitens [Kni]; Chlorokybus atmophyticus [Cat]; Mesostigma viride [Mvi]) and chlorophyte (Volvox carteri (Vca); Chlamydomonas reinhardtii [Cre]; Ostreococcus lucimarinus [Olu]) algae.

Our analysis revealed that $A$. thaliana was the species with more proteins containing either canonical or non-canonical RBR-binding motifs (Fig. 1, Table S1), with 8 out of 23 RdDM-related proteins analysed (DCL3, DRD1, DRM2, NRPD2, SUVR2, CMT2, JMJ14 and NRPE1). DCL3 orthologs showed the highest level of conservation for canonical and non-canonical LXCXE SLiMs among the species analyzed as they are absolutely conserved in tracheophytes, with the only exception of $G$. montanum. Interestingly, while DCL3 in $M$. polymorpha bears a canonical LXCXE SLiM, DCL3 orthologs in other bryophytes specifically $S$. fallax, P. patens and $A$. agrestis contain LXCxE-like SLiMs. From the seven charophyte algae species analysed, 3 of them contain canonical RBR-binding motif ( $P$. margaritaceum $M$. endlicheranium, $K$ nitens) while $S$. muscicola contains an LxCXE SLiM (Fig. 1, Table S1). Although the LXCXE SLiM is highly conserved along DCL3 orthologs, it is difficult to determine if the canonical or the non-canonical motif is the ancestral one.

DRD1 orthologues showed the presence of the LXCXE SLiM in a patchy pattern along the plant lineages analyzed. The presence of the LXCXE SLiM in DRD1 orthologues is less conserved than in DCL3 orthologues since we were not able to find LXCXE or LXCXE-like SLiMs in any of the algae species analyzed, however it is present in Marchantia, Anthoceros, and Ceratopteris DRD1 orthologs (Fig. 1, TableS1). The presence of the LXCXE SLiM is even less conserved in DRM2 orthologs than in DRD1, with only two DRM2 orthologs from Arabidopsis and Boechera exhibiting a canonical SLiM and non-canonical LXCXE SLiMs present in 
234 Pinus, Sphagnum, Antoceros and Mesostigma. In the case of the subunits of POLIV 235 and POLV, we expanded a presence-absence analysis along the plant phylogeny, 236 similar to that reported previously by Huang et al. (2015). We found that NRPE1, 237 NRPD1 and NRPD2 showed the presence of both canonical and non-canonical 238 LXCXE SLiMs in diverse species, among these 3 proteins we found that NRPE1 is 239 the one with more species containing either canonical or non-canonical RBR-binding 240 SLiM (Fig. 1, TableS1). While Arabidopsis NRPD1 does not contain an LXCXE SLiM, 241 P. taeda NRPD1 ortholog contains a canonical LXCXE SLiM and orthologs from S. 242 viridis, $P$. abies, maize and tomato bear a non-canonical LXCXE SLiM. We observed 243 the presence of canonical LxCXE SLiMs in NRPE1 from charophyte to flowering 244 plants (P. margaritaceum, M. polymorpha, P. abies, P. taeda, Z. mays and O. sativa) 245 and non-canonical LXCXE SLiMs in NRPE1 orthologs from $C$. richardii, $A$. agrestis, 246 P. patens, G. montanum, S. viridis and A. thaliana. The presence of canonical and 247 noncanonical LXCXE SLiMs involved in RBR-binding in the POLIV and POLV largest 248 subunits (NRPD1 and NRPE1, respectively) and the shared second largest subunit 249 (NRPD/E2) strongly suggests that a new layer of regulation of the RdDM pathway 250 mediated by RBR is present in land plants. 


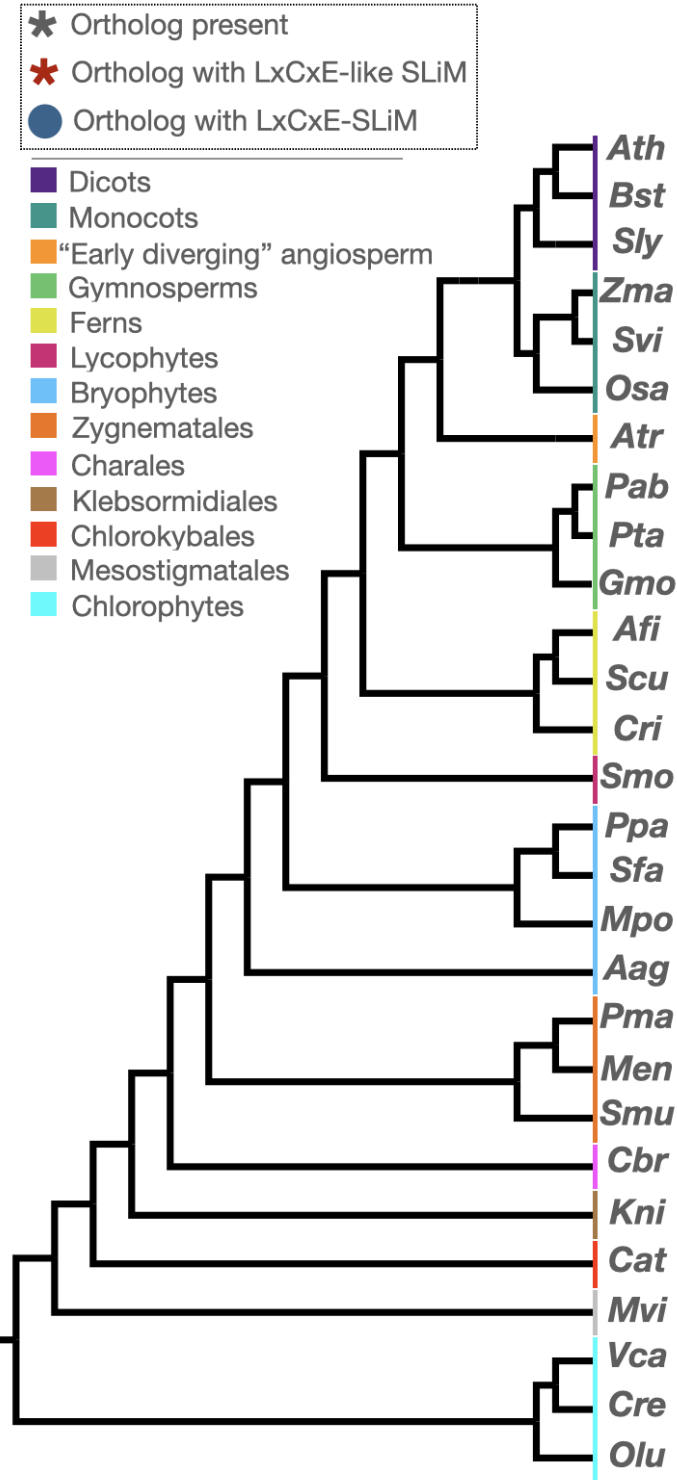

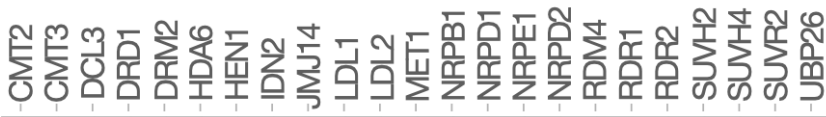
O*O $* * * 0 * * * * * * 0 * * * * * 0 *$ **O* $* * * * 0 * * * * * * 0 * * * * * * *$ **O************O* $* * * * *$

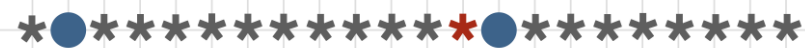
**OO*** *t********0*****

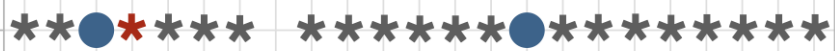
$* * 0 * * 0 * * * * * * * * * * * * * * *$ $* * 0 * * * * * * * * * * * 0 * * 0 * * *$

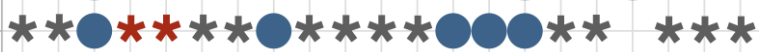
** $* * * * * * * * * * * * * * * 0 * * 00$ *O $* * * * * * * 0 * t * 0 * * * * * 0 *$ * $0 * * * * * * * * * * * * *$ * O $* * * * * * * * * * * * * * * * * *$ t*t*t $* t * t * t * t \quad t * t * t *$ $* * * * * * * * * * * * * * * * * * * * * *$

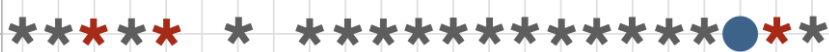

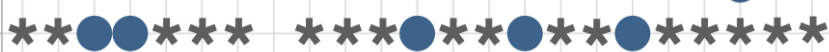
$* * * 0 * t * * * * 0 * * * * * * * * *$

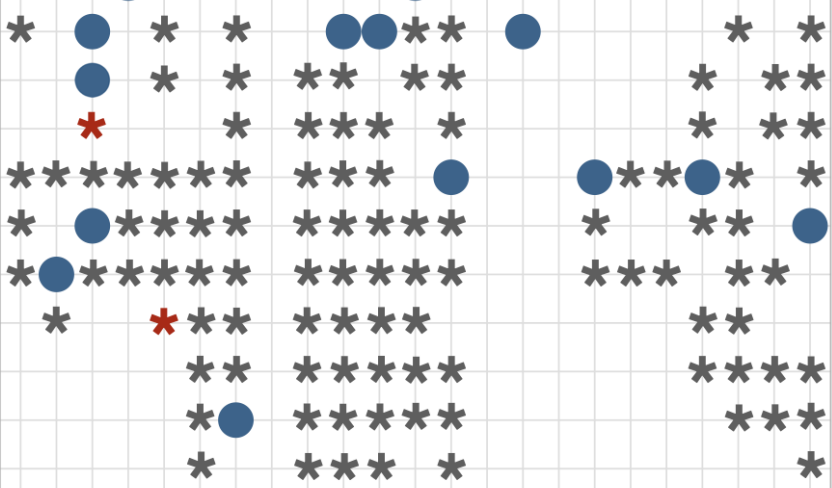

Fig. 1. Phylogenetic conservation of canonical (solid blue circle) and non-canonical (red asterisk) LxCxE SLiMs in orthologs of the RdDM pathway in representative species along 254 Viridiplantae.

Based on their conservation patterns we selected a group of proteins to test for protein-protein interactions with RBR. We generated constructs using amplified coding sequences (CDS) of DRM2, DRD1 and SUVR2 from Arabidopsis for $\mathrm{Y} 2 \mathrm{H}$ assays, in order to test if they interact with RBR (previously cloned in pDEST32 and used in Cruz-Ramirez et al., 2012). Our results showed that SUVR2 strongly interacts with RBR when quantified and compared with other partners and controls (Fig.2 a, b) but DRD1 and DRM2 showed weak interaction. The previously described 
$\mathrm{Y} 2 \mathrm{H}$ results prompted us to confirm, using a semi in vivo system, DRD1-RBR and DRM2-RBR interactions by Bimolecular Fluorescence Complementation (BiFC) assays. We found that YFP nuclear signal is clear and evident in Arabidopsis mesophyll protoplasts, confirming that DRD1 and DRM2 do interact with RBR. We also found that the $M$. polymorpha DCL3 ortholog interacts with both Arabidopsis and $M$. polymorpha RBRs by $\mathrm{Y} 2 \mathrm{H}$ assays (León-Ruiz \& Cruz-Ramirez, in preparation). Further experimental work is required to confirm PPIs between RBR and other RdDM-related proteins including NRPD1, NRPE1 and NRPD/E2 but it is important to consider that regulation by RBR can go beyond its direct interactors, for example it can affect other PPIs indirectly as documented in the IntAct Molecular Interactions Database from EMBL-EBI: DRM2 establishes 12 PPIs, from which at least 4 are direct interactions with members of the RdDM pathway, such as RDM1, AGO4, AGO9, and ZOP1

(Fig.4d. (https://www.ebi.ac.uk/intact/interactions?conversationContext=4). Taken together, our results indicate that the evolutionary conservation of LxCXE SLiMs among components of the RdDM pathway is consistent with our experimentally validated interactions with RBR in the cases of DRD1, DRM2 and SUVR2.

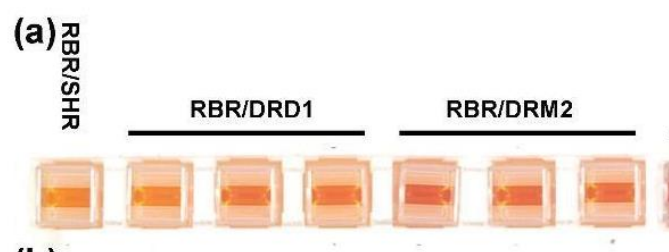

(b)

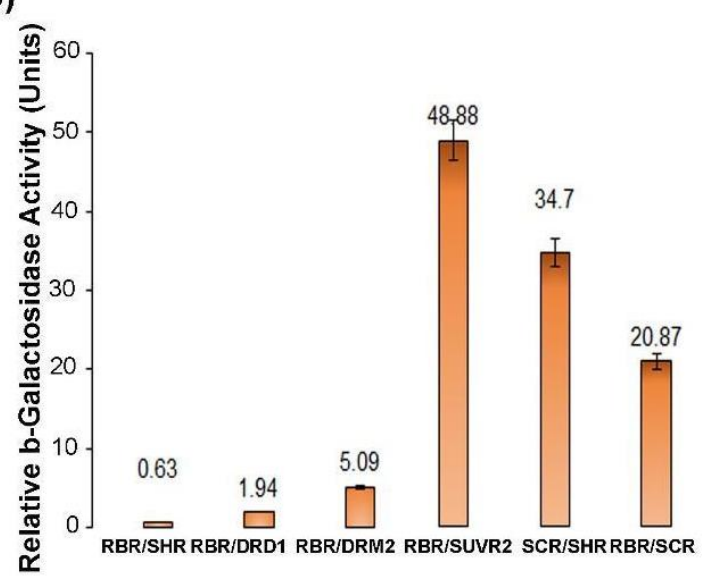

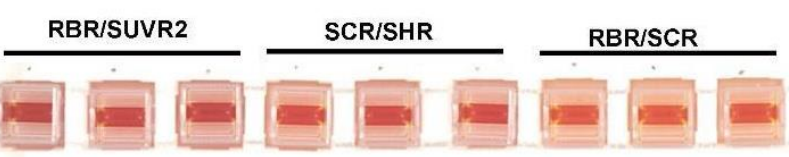

(c)

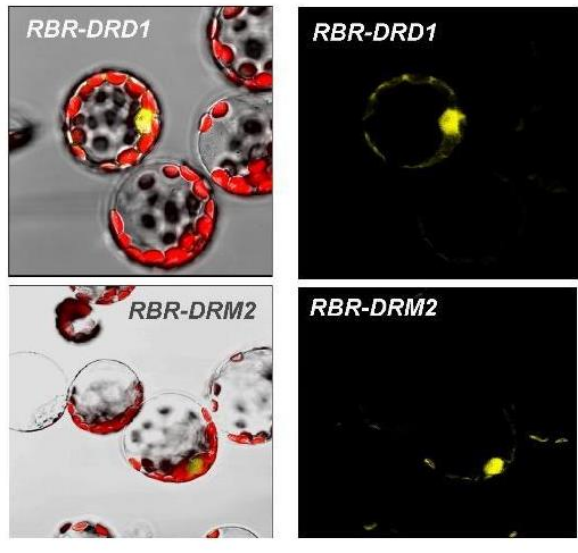

Fig. 2. (a) Yeast two-hybrid analyses showing b-gal colorimetric reaction and its quantitation, in (b), for diverse proteins of the RdDM pathway and RBR. SCR-RBR and SCR-SHR combinations are positive controls, and RBR-SHR is the negative control (Cruz-Ramírez, etal, 2013) (c) RBR-DRD1 and RBR-DRM2 binding by BiFC in Arabidopsis mesophyll protoplasts. 
RdDM and RBR loss-of-function mutants show similar developmental alterations

291 It has been shown that loss of function mutants in members of the RdDM pathway 292 show phenotypes in diverse developmental processes and stages of Arabidopsis 293 (He et al., 2009; reviewed in Matzke et al., 2015; Mendes et al., 2020).

294 In addition to physically interacting, RBR and DRM2 protein fusions 295 (pRBR::RBR:CFP, pDRM2::DRM2:GFP) have quite similar expression patterns as 296 both proteins are present in every cell of the RAM (Fig.3 a,b). Since RBR has been 297 shown to regulate stem cells and QC divisions in the Arabidopsis RAM (Cruz298 Ramirez et al., 2012; Cruz-Ramirez, et al., 2013), we wondered if loss of function 299 (LOF) mutants, in tested and putative interactors, in genes of the RdDM pathways 300 may display similar phenotypes to those in RBR LOF lines. Therefore, we analyzed 301 root development of $12 \mathrm{dpg}$ (days post germination) seedlings of the $302 \mathrm{drm} 1 ; \mathrm{drm} 2 ; \mathrm{cmt3}$ triple mutant and the nrpd2a;nrpd2b double mutant and observed 303 that primary root development in these mutants is affected. Although the phenotype 304 is variable among seedlings from mild to severe, they all exhibit a shorter 305 meristematic zone (Fig.S1 a, b, c). We analyzed in detail the organization of the RAM 306 and root stem cell niche (RSCN) of drm1;drm2;cmt3 and nrpd2a;nrpd2b $10 \mathrm{dpg}$ 307 seedlings and observed that roots from both mutant lines showed a disorganized 308 RAM and defects in the columella region relative to wild-type seedlings (Fig.3). In 309 addition, the loss of function in RBR causes QC divisions, extra stem cells and 310 aberrant divisions and alterations in the columella region, as revealed for the 311 analysis of the 35S::AmiGO-RBR RAM (Fig.3 e, f). Columella phenotypes observed 312 in RdDM and RBR loss-of-function mutants, shown in Fig.3 and Fig.S1, are 313 consistent with findings in this tissue by Kawakatsu et al. (2016), who reported that 314 the Arabidopsis columella root cap genome is hypermethylated and transcripts 315 encoding RdDM factors, as well as 24-nt small RNAs (smRNAs), are more abundant 316 in this tissue than any other root cell type. 

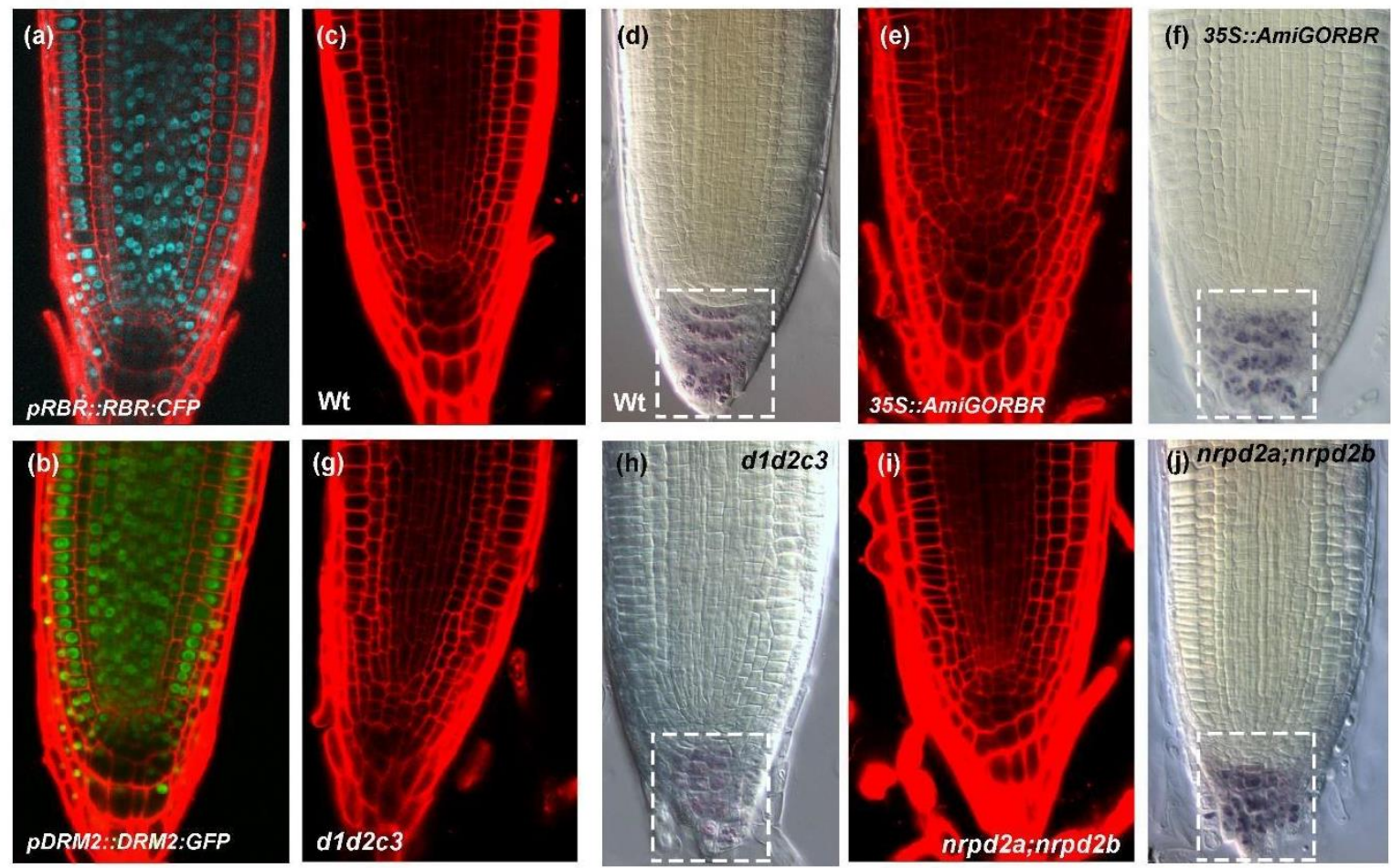

Fig. 3. Longitudinal root sections of $10 \mathrm{dpg}$ seedlings imaged by confocal laser scanning microscope (CLSM) (a), (b), (c), (e), (g), (i), and Nomarski optics of lugol-stained roots (d), (f), (h) and (j). Panels (a) and (b) show the expression patterns of $p R B R:: R B R: C F P$ and pDRM2::DRM2:GFP. (c) confocal and (d) Nomarski optics images showing root apical meristem (RAM) and root stem cell niche (RSCN) organization in Col-0 (WT) seedlings. (e) Confocal and ( $\mathrm{f}$ ) Nomarski images of 35S::AmiGO-RBR seedlings showing alterations in the RAM and RSCN. (g) Confocal and (h) Nomarski images of drm1;drm2;cmt3 (d1d2c3) triple mutant seedlings showing alterations in the RAM and the RSCN. (i) Confocal and (j) Nomarski images of nrpd2a;nrpd2b double mutant seedlings showing phenotypes in the RAM and RSCN, dotted squares highlight the Columella region.

\section{RdDM and SUVR2 targets are up-regulated in the AmiGO-RBR background}

SUVR2 silences a subset of RdDM target loci, as well as RdDM-independent targets (Hang et al., 2014). Well-known targets of RdDM include TEs from the solo LTR (SLTR) and AtGP1 LTR families and genes such as SUPPRESSOR OF drm1 drm2 cmt3 $(S D C)$ and it has been shown that at SDC and ERT7 loci, the suvr2 loss-offunction mutants display a synergistic phenotype with mutants in key genes of the RdDM pathway, which suggests that at these loci SUVR2 might exert silencing through a pathway which is partially independent of RdDM (Hang et al., 2014). Our data indicates that SUVR2, DRM2 and DRD1 bind in vitro to RBR and based on the presence of the LxCxE SLiM other RdDM components like NRPD1, NRPE1 and DCL3 could also potentially bind to RBR. Therefore, we wondered if the silencing of 
background. To answer such question, we is olated total RNA of 12-days-old wildtype and 35S::AmiGO-RBR seedlings and performed qRT-PCR assays using previously-reported primers for SDC, AtGP1, solo LTR (SLTR), AT1TE51360 (AT1TE), AT2TE78930 (AT2TE), ERT7, ERT9, ERT12, and ERT14. Our results showed that all tested loci are either moderately or strongly up-regulated in the RBR loss-of-function background relative to the wild-type control (Fig.4a). It has been shown that ERT9 transcripts are not de-repressed in the suvr2 mutant background, suggesting that RBR might influence DRM2 and SUVR2 targets independently. Overall, these results indicate that RBR acts repressing RdDM and SUVR2 transposable elements targets. Whether this action depends on RBR protein-protein interaction with DRM2, SUVR2 or DRD1 remains to be answered in future studies.

The RdDM pathway methylates not only TE loci, but also hundreds of proteincoding genes (Jha \& Shanka, 2014). Since RBR also has hundreds of targets, predicted by Chip-Seq (Bouyer et al., 2018), we explored a potential overlap between the 4,431 DRM2 methylation targets proposed by Jha and Shankar (2014) and the 1,729 RBR target genes predicted by Bouyer et al., (2018). We found that 245 target genes are shared between RBR and DRM2 (Fig.4b). Among the 245 shared target genes we found several interesting ones (Fig. 4c). We highlighted those that encode transcriptional regulators such as POLAR LOCALIZATION DURING ASYMMETRIC DIVISION AND REDISTRIBUTION (POLAR), AGAMOUS LIKE 15, NAC15, INDOLE-3-ACETIC ACID INDUCIBLE 5 (IAA5) and MADS AFFECTING FLOWERING 3 (MAF3). Another important transcription factor that has been shown to act downstream RBR is WUSCHEL. In rbr1-2 mutants, supernumerary megaspore mother cells (MMCs) are formed, a phenotype that correlates with WUS transcriptional deregulation Zhao et al. (2017). Indeed these authors demonstrate that RBR binds to a specific region on the WUS promoter. It has also been shown that in the $d r m 1 ; d r m 2 ; c m t 3$ WUS transcription is de-repressed during root regeneration and that two non-CG sites in the promoter of this gene might be related to WUS silencing in Arabidopsis roots (Shermer et al., 2015). Recently Mendes et al. (2020) showed that drm1;drm2 double mutants develop multiple MMCs, a phenotype also described for other mutants in key genes of the RdDM pathway, such as rdr6 and ago9 (Olmedo-Monfil et al., 2010). We also found that genes related to DNA integrity, DNA replication and cell cycle are common targets of RBR and DRM2, such as RAD51, PROLIFERATING CELL NUCLEAR ANTIGEN 2 (PCNA2), MINICHROMOSOME MAINTENANCE 7/PROLIFERA (MCM7), MS1, MEDIATOR 19B (MED19B), CYCLIN A1;1 (CYCA1;1), CELL DIVISION CONTROL $6 B$ (CDC6B) and CYCLIN B2;4 (CYCB2;4) (Table S2). The putative function of RBR and DRM2 acting on the silencing of genes involved in cell cycle progression, which are normally expressed only in root meristematic cells, such as CDC6B or CYCA1;1, correlate with some of the root phenotypes reported in Fig. 3 and Fig.S1. However, root phenotypes in RdDM mutants are not similar in all cases, and such contrasting phenotypes may be caused by the deregulation of hundreds of genes with diverse cellular functions. 
385 Overall, this study uncovers novel mechanisms for RBR function in transcriptional 386 silencing through interacting with components of the RdDM pathway and opens 387 novel working hypotheses for diverse potential RBR-RdDM interactions (Fig. 4d), 388 including the RBR-DRM2 complex, regulating TEs and interesting lineage-specific 389 transcription factors. 
(a)

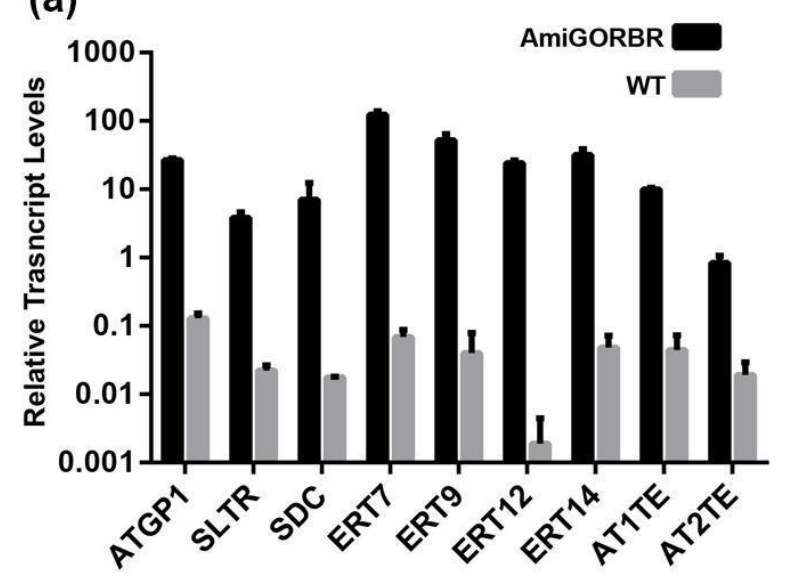

(b)

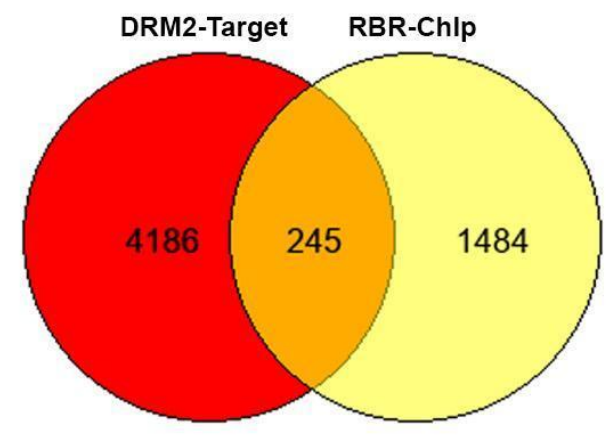

(c)

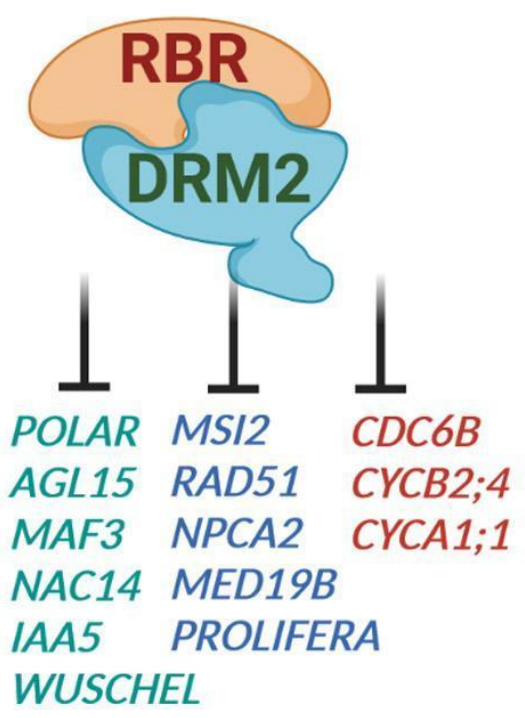

(d)

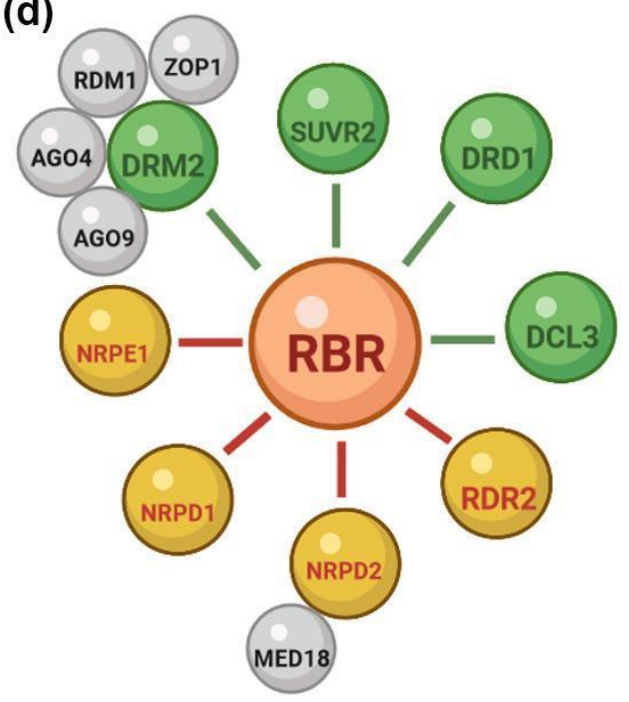

Fig. 4. (a) Transcript levels revealed by qRT-PCR of RdDM targets in the AmiGO-RBR mutant background vs the control, (b) RBR-ChIP and DRM2-mediated DNA methylation common targets, (c) Key examples of RBR-DRM2 common targets, (d) RdDM proteins that have been validated as RBR direct interactors (green lines) and those that are putatively binding RBR (red lines), gray balloons are PPIs with DRM2 reported in the IntAct database 
403 We wish to thank Vicki Chandler, Steve Jacobsen, Fred Berger and Pauline Jullien

404 for sharing published plant materials. We also thank Dr. Juan Caballero-Pérez for 405 initial advice on bioinformatics. J L-R (CVU 858608) was supported by Consejo 406 Nacional de Ciencia y Tecnología (CONACYT) with a PhD Fellowship. A C-R was 407 supported by EMBO-ALTF 1114-2006 and CONACYT 000000000092916 grants. M 408 A-V was supported by Consejo Nacional de Ciencia y Tecnología (CONACYT) 409 grants 158550 and A1-S-38383 and Newton Fund of the Royal Society grant 410 NA150181.

\section{Author contributions}

413 Conceived the project: $\mathrm{A} \mathrm{C}-\mathrm{R}$ and $\mathrm{M} \mathrm{A}-\mathrm{V}$. Performed wetlab and in silico 414 experiments: A C-R, A E-C, J L-R, I B. Analyzed the data: A C-R, M A-V, B S, I B, A 415 E-C and J L-R. Contributed reagents and equipment: B S, M A-V, A C-R. Wrote the 416 manuscript with inputs from all coauthors: AC-R and MA-V.

\section{ORCID}

418 A C-R https://orcid.org/0000-0001-9973-5784

419 M A-V https://orcid.org/0000-0002-9435-0049

420 B S https://orcid.org/0000-0001-5400-9578

421 I B https://orcid.org/0000-0001-8003-3782

422 A E-C https://orcid.org/0000-0002-5421-1503 


\section{References}

428

429

430

431

432

433

434

435

436

437

438

439

440

441

442

443

444

445

446

447

448

449

450

451

452

453

454

455

456

457

458

459

460

461

462

463

464 8374-8381. 14(11):e1007797. 16(10):e1009034.

Ausin I, Greenberg MV, Simanshu DK, Hale CJ, Vashisht AA, Simon SA, Lee TF, Feng S, et al. 2012 . INVOLVED IN DENOVO 2-containing complex involved in RNA-directed DNA methylation in Arabidopsis. Proc. Natl. Acad. Sci. USA 109

Borges F, Martienssen RA. 2015. The expanding world of small RNAs in plants. Nat Rev Mol Cell Biol. 16(12):727-41.

Bouyer D, Heese M, Chen P, Harashima H, Roudier F, Grüttner C, Schnittger A. 2018. Genome-wide identification of RETINOBLASTOMA RELATED 1 binding sites in Arabidopsis reveals novel DNA damage regulators. PLoS Genet.

Calo E, Quintero-Estades JA, Danielian PS, Nedelcu S, Berman SD, Lees JA. 2010. $\mathrm{Rb}$ regulates fate choice and lineage commitment in vivo. Nature. 26;466(7310):1110-1114.

Cruz-Ramírez A, López-Bucio J, Ramírez-Pimentel G, Zurita-Silva A, SánchezCalderon L, Ramírez-Chávez E, González-Ortega E, Herrera-Estrella L. 2004. The xipotl mutant of Arabidopsis reveals a critical role for phospholipid metabolism in root system development and epidermal cell integrity. Plant Cell. 2004 16(8):2020-2034.

Cruz-Ramírez A, Díaz-Triviño S, Blilou I, Grieneisen VA, Sozzani R, Zamioudis C, Miskolczi P, Nieuwland J, Benjamins R, Dhonukshe P, Caballero-Pérez J, Horvath $B$, Long $Y$, Mähönen AP, Zhang $H, X u$ J, Murray JA, Benfey PN, Bako L, Marée AF, Scheres B. 2012. A bistable circuit involving SCARECROW-RETINOBLASTOMA integrates cues to inform asymmetric stem cell division. Cell. 31;150(5):1002-1015.

Erdmann RM, Picard CL. 2020. RNA-directed DNA Methylation. PLoS Genet.

Haag JR, Pikaard CS. 2011. Multisubunit RNA polymerases IV and V: purveyors of non-coding RNA for plant gene silencing. Nat Rev Mol Cell Biol. 12(8):483-92.

Han YF, Dou K, Ma ZY, Zhang SW, Huang HW, Li L, Cai T, Chen S, Zhu JK, He XJ. 2014. SUVR2 is involved in transcriptional gene silencing by associating with SNF2related chromatin-remodeling proteins in Arabidopsis. Cell Res. 24(12):1445-1465.

He XJ, Hsu YF, Zhu S, Liu HL, Pontes O, Zhu J, Cui X, Wang CS, Zhu JK. 2009. A conserved transcriptional regulator is required for RNA-directed DNA methylation and plant development. Genes Dev. 1;23(23):2717-22.

Huang Y, Kendall T, Forsythe ES, Dorantes-Acosta A, Li S, Caballero-Pérez J, Chen X, Arteaga-Vázquez M, Beilstein MA, Mosher RA. 2015. Ancient Origin and Recent Innovations of RNA Polymerase IV and V. Mol Biol Evol. 32(7):1788-1799. 
465

466

467

468

469

470

471

472

473

474

475

476

477

478

479

Jha A, Shankar R. 2014. MiRNAting control of DNA methylation. J Biosci. 39(3):365380.

Johnston AJ, Matveeva E, Kirioukhova O, Grossniklaus U, Gruissem W. 2008. A dynamic reciprocal RBR-PRC2 regulatory circuit controls Arabidopsis gametophyte development. Curr Biol. 18(21):1680-1686.

Jullien PE, Mosquna A, Ingouff M, Sakata T, Ohad N, Berger F. 2008. Retinoblastoma and its binding partner MSI1 control imprinting in Arabidopsis. PLOS Biol. 12;6(8):e194.

Jullien PE, Susaki D, Yelagandula R, Higashiyama T, Berger F. 2012. DNA methylation dynamics during sexual reproduction in Arabidopsis thaliana. Curr Biol. 9;22(19):1825-1830.

Kawakatsu T, Stuart T, Valdes M, Breakfield N, Schmitz RJ, Nery JR, Urich MA, Han X, Lister R, Benfey PN, Ecker JR. 2016. Unique cell-type-specific patterns of DNA methylation in the root meristem. Nat Plants. 29;2(5):16058.

Law JA, Jacobsen SE. 2010. Establishing, maintaining and modifying DNA methylation patterns in plants and animals. Nat Rev Genet. 11(3):204-20.

Matos JL, Lau OS, Hachez C, Cruz-Ramírez A, Scheres B, Bergmann DC. 2014. Irreversible fate commitment in the Arabidopsis stomatal lineage requires a FAMA and RETINOBLASTOMA-RELATED module. Elife. 10;3:e03271.

Matzke MA, Mosher RA. 2014. RNA-directed DNA methylation: an epigenetic pathway of increasing complexity. Nat Rev Genet. 15(6):394-408.

Matzke MA, Kanno T, Matzke AJ. RNA-Directed DNA Methylation: The Evolution of a Complex Epigenetic Pathway in Flowering Plants. 2015. Annu Rev Plant Biol. 2015;66:243-67.

Mendes MA, Petrella R, Cucinotta M, Vignati E, Gatti S, Pinto SC, Bird DC, Gregis V, Dickinson H, Tucker MR, Colombo L. 2020. The RNA-dependent DNA methylation pathway is required to restrict SPOROCYTELESS/NOZZLE expression to specify a single female germ cell precursor in Arabidopsis. Development. 147(23):dev194274.

Oliva M, Butenko Y, Hsieh TF, Hakim O, Katz A, Smorodinsky NI, Michaeli D, Fischer RL, Ohad N. 2016. FIE, a nuclear PRC2 protein, forms cytoplasmic complexes in Arabidopsis thaliana. J Exp Bot. 67(21):6111-6123.

Olmedo-Monfil V, Durán-Figueroa N, Arteaga-Vázquez M, Demesa-Arévalo E, Autran D, Grimanelli D, Slotkin RK, Martienssen RA, Vielle-Calzada JP. 2010. Control of female gamete formation by a small RNA pathway in Arabidopsis. Nature. 464(7288):628-32. 
501

502

503

504

505

506

507

508

509

510

511

512

513

514

515

516

517

518

519

520

521

522

523

524

525

526

527

528

529

530

531

532

533

534

535

536

537

538
Ötvös K, Miskolczi P, Marhavý P, Cruz-Ramírez A, Benková E, Robert S, Bakó L. 2021. Pickle Recruits Retinoblastoma Related 1 to Control Lateral Root Formation in Arabidopsis. Int J Mol Sci. 8;22(8):3862.

Shemer O, Landau U, Candela H, Zemach A, Eshed Williams L. 2015. Competency for shoot regeneration from Arabidopsis root explants is regulated by DNA methylation. Plant Sci. 238:251-261.

Trujillo JT, Seetharam AS, Hufford MB, Beilstein MA, Mosher RA. 2018. Evidence for a Unique DNA-Dependent RNA Polymerase in Cereal Crops. Mol Biol Evol. 35(10):2454-2462.

Willemsen V, Wolkenfelt H, de Vrieze G, Weisbeek P, Scheres B. 1998. The HOBBIT gene is required for formation of the root meristem in the Arabidopsis embryo. Development. 125(3):521-531.

Zhang Y, Shi C, Fu W, Gu X, Qi Z, Xu W, Xia G. Arabidopsis MED18 Interaction With RNA Pol IV and V Subunit NRPD2a in Transcriptional Regulation of Plant Immune Responses. 2021. Front Plant Sci. 6;12:692036.

Zhao C, Lasses T, Bako L, Kong D, Zhao B, Chanda B, Bombarely A, Cruz-Ramírez A, Scheres B, Brunner AM, Beers EP. 2017. XYLEM NAC DOMAIN1, an angiosperm NAC transcription factor, inhibits xylem differentiation through conserved motifs that interact with RETINOBLASTOMA-RELATED. New Phytol. 216(1):76-89.

Zhao X, Bramsiepe J, Van Durme M, Komaki S, Prusicki MA, Maruyama D, Forner J, Medzihradszky A, Wijnker E, Harashima H, Lu Y, Schmidt A, Guthörl D, Logroño RS, Guan Y, Pochon G, Grossniklaus U, Laux T, Higashiyama T, Lohmann JU, Nowack MK, Schnittger A. 2017. RETINOBLASTOMA RELATED1 mediates germline entry in Arabidopsis. Science. 28;356(6336):eaaf6532.

Zhou W, Lozano-Torres JL, Blilou I, Zhang X, Zhai Q, Smant G, Li C, Scheres B. 2019. A Jasmonate Signaling Network Activates Root Stem Cells and Promotes Regeneration. Cell. 177(4):942-956e. 
Fig.S1. RAM phenotypes in RdDM mutants.

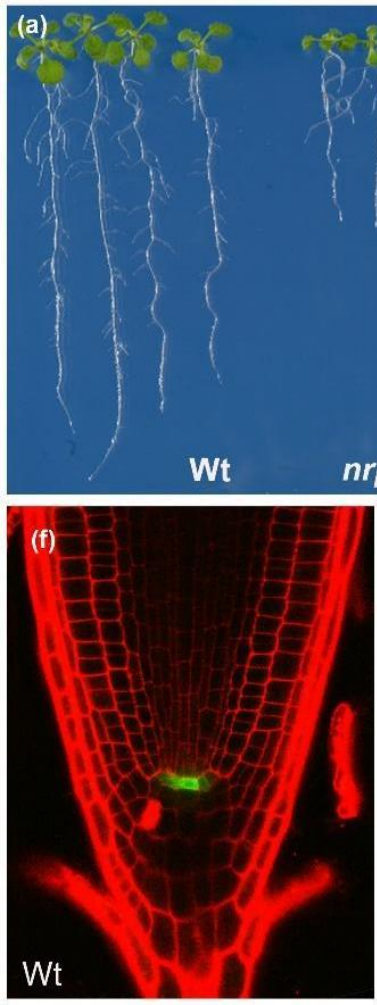

\section{(b)}

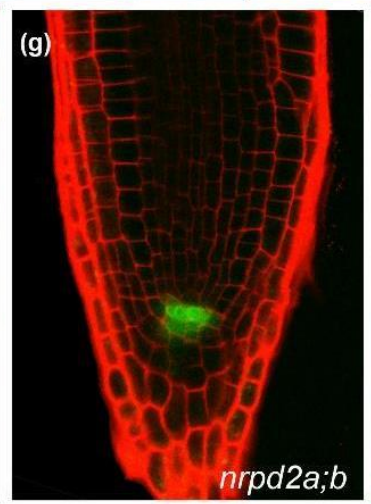

(c)
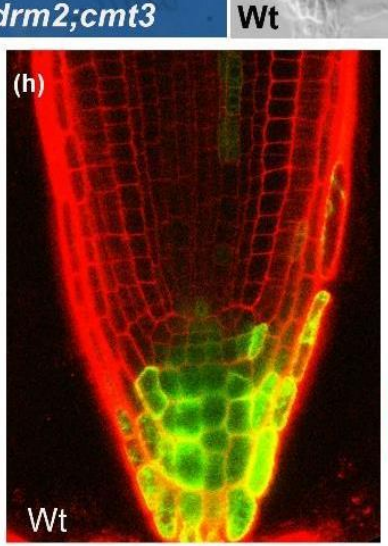

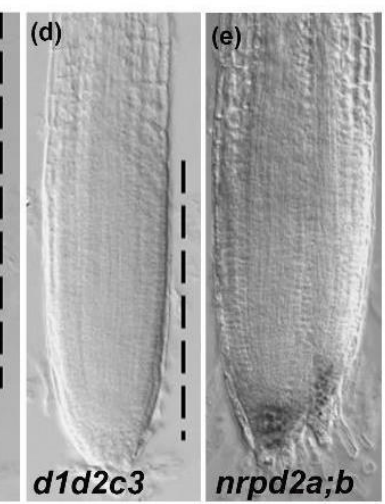

d1d2c3

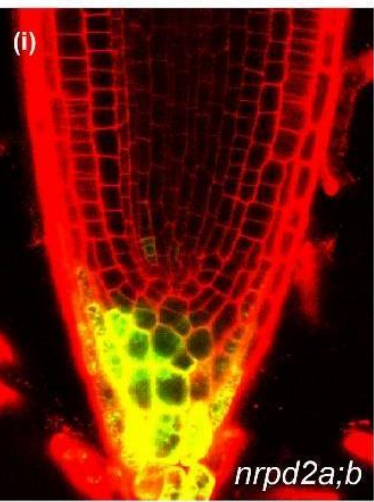

545 Fig._S1. Root and shoot phenotypes, recorded with stereomicroscope, of 10 days post 546 germination (dpg) seedlings of wild-type (a), double and triple mutants in RdDMproteins (a), 547 (b). Nomarski optics for RAM phenotypes of $d r m 1 ; d r m 2 ; c m t 3$ and nrpd2a;nrpd2bmutant(d), 548 (e) and wild-type (c) roots of $10 \mathrm{dpg}$ seedlings. Longitudinal root sections of $10 \mathrm{dpg}$ seedlings 549 by confocal laser scanning microscope (CLSM) of pWOX5::GFP and TCS::GFPtransgenes in WT (f), (h) and nrpd2a;nrpd2b (g), (i) backgrounds, respectively. 